\title{
Accessing the gluon Sivers function at a future electron-ion collider
}

\author{
L. Zheng, ${ }^{1,2,{ }^{*}}$ E. C. Aschenauer, ${ }^{3, \dagger}$ J. H. Lee, ${ }^{3, \$}$ Bo-Wen Xiao, ${ }^{2, \S}$ and Zhong-Bao Yin ${ }^{2, \|}$ \\ ${ }^{1}$ School of Mathematics and Physics, China University of Geosciences (Wuhan), Wuhan 430074, China \\ ${ }^{2}$ Key Laboratory of Quark and Lepton Physics (MOE) and Institute of Particle Physics, \\ Central China Normal University, Wuhan 430079, China \\ ${ }^{3}$ Physics Department, Brookhaven National Laboratory, Upton, New York 11973, USA
}

(Received 15 May 2018; published 13 August 2018)

\begin{abstract}
In this work, we present a systematic study on the feasibility of probing the largely unexplored transverse-momentum-dependent gluon Sivers function (GSF) in open charm production, and high- $p_{T}$ charged dihadron and dijet production at a future high-energy, high-luminosity electron-ion collider (EIC). The Sivers function is a measure for the anisotropy of the parton distributions in momentum space inside a transversely polarized nucleon. It is proposed that it can be studied through single spin asymmetries in the photon-gluon fusion subprocess in electron-proton collisions at the EIC. Using a well-tuned Monte Carlo model for deep inelastic scattering, we estimate the possible constraints of the GSF from the future EIC data. A comparison of all the accessible measurements illustrates that the dijet channel is the most promising way to constrain the magnitude of the GSF over a wide kinematic range.
\end{abstract}

DOI: 10.1103/PhysRevD.98.034011

\section{INTRODUCTION}

In recent years, an important topic in hadron physics has been the exploration of the $2+1$-dimensional partonic structure of nucleons by including information on the internal parton transverse momentum- and coordinate-space distributions. These extensions have significantly broadened our understanding of the nucleon structure compared to the one-dimensional picture in the longitudinal momentum space. The transverse momentum structure of nucleons is encoded in the transverse-momentum-dependent parton distribution functions (TMDs) [1], which contain information on both the longitudinal momentum fraction $x$ and the transverse (sometimes called intrinsic) motion $k_{\perp}$ of quarks and gluons inside a fast-moving nucleon.

When including spin degrees of freedom, TMDs link information on the intrinsic spin of a parton $\left(s_{q, g}\right)$ and their transverse motion $\left(k_{\perp}^{q, g}\right)$ to the spin direction of the parent nucleon. At leading twist the most general spin-dependent TMD can be denoted by $f_{1}^{q, g}\left(x, k_{\perp}^{q, g} ; s_{q, g}, S\right)$. At leading order, there are eight such combinations, yielding eight

\footnotetext{
*zhengliang@cug.edu.cn

†elke@bnl.gov

\#hlee@bnl.gov

bxiao@mail.ccnu.edu.cn

zbyin@mail.ccnu.edu.cn
}

Published by the American Physical Society under the terms of the Creative Commons Attribution 4.0 International license. Further distribution of this work must maintain attribution to the author(s) and the published article's title, journal citation, and DOI. Funded by SCOAP ${ }^{3}$. independent TMDs [2]. The Sivers function $f_{1 T}^{\perp}$ [3], which encapsulates the correlations between a parton's transverse momentum inside the proton and the spin of the proton, has received the widest attention both phenomenologically and experimentally among all TMDs. It was found that the Sivers function is not universal in hard-scattering processes [4], which has its physical origin in the rescattering of a parton in the color field of the remnant of the polarized proton [5]. Proving experimentally the process dependence of the Sivers function is a very important test of the nonAbelian nature of quantum chromodynamics (QCD) in TMD factorization.

Experimentally, the quark Sivers function has been measured in semi-inclusive deep inelastic scattering (SIDIS) by the HERMES, COMPASS, and JLab Hall A collaborations [6-8]. However, due to the limited statistics precision and the narrow kinematic coverage of the SIDIS data, only the valence quark Sivers function at moderate to high $x$ could be constrained in phenomenological fits [9]. The quark Sivers function has also been studied in polarized proton-proton collisions by the STAR and PHENIX collaborations at the Relativistic Heavy Ion Collider (RHIC) [10,11]. There are first indications both from STAR through the $W$-boson measurement [12] and COMPASS in Drell-Yan (DY) production [13] for the nonuniversality of the Sivers function [5] if measured in hadron-hadron collisions or SIDIS, but the still challenging statistical precision limits a definite conclusion. Both STAR and COMPASS will soon increase the statistical precision of these measurements by including recent highstatistics data. At the future high-energy, high-luminosity 
electron-ion collider (EIC) [14], the quark Sivers function can be well constrained over a very wide kinematic range $\left(x, Q^{2}, z\right.$ and $\left.p_{T}\right)$ in SIDIS with exquisite precision. It has been systematically investigated in the one- and twohadron final states in Ref. [15] with a modified PYTHIA event generator that includes the quark Sivers effect.

The gluon Sivers function (GSF), on the other hand, is barely known at the present time [16]. Presently, the major theoretical constraint for the GSF comes from the Burkardt sum rule [17], which requires the total transverse momentum of all partons in a transversely polarized nucleon to vanish. The only direct constraint of the GSF comes from the left-right asymmetry $A_{N}$ data in $p^{\uparrow} p \rightarrow \pi^{0} X$ within the so-called TMD generalized parton model (GPM) framework [18]. This analysis found that the gluon Sivers function is not large [19]. However, the gluon Sivers function obtained in the GPM may differ from the gluon Sivers function in the TMD framework [16]. At this moment the only experimental constraint on the gluon Sivers function in the TMD framework comes from the recent SIDIS measurement of high- $p_{T}$ hadron pairs off transversely polarized deuterons and protons at COMPASS [20]. This analysis found that the gluon Sivers asymmetry is negative at large $x_{B}$ within statistical uncertainties. Interestingly, this finding is in qualitative agreement with results from the calculation based on the light-cone spectator model [21].

Accounting for the different gauge-link structures involved in deep-inelastic scattering (DIS) and hadronic collisions, the gluon Sivers function is expected to be process dependent. A test of this nonuniversality of the gluon Sivers function is of equal importance as for the quarks and is currently not validated. Similar to the sign change of the quark Sivers function, the gluon Sivers function accessed in $e p^{\uparrow} \rightarrow e^{\prime} c \bar{c} X$ is also predicted to be related to that in $p^{\uparrow} p \rightarrow \gamma \gamma X$ by an overall sign change $f_{1 T}^{g}\left[e p^{\uparrow} \rightarrow e^{\prime} c \bar{c} X\right]=$ $-f_{1 T}^{g}\left[p^{\uparrow} p \rightarrow \gamma \gamma X\right]$ as shown in Ref. [22]. Consequently, the study of the gluon Sivers function at an EIC will provide a unique test of the fundamental nonperturbative QCD effects through complementary information on the proposed gluon Sivers function observables at the RHIC and LHC [23,24]. In addition, as pointed out in Ref. [25], there are two different types of gluon TMDs, namely, the WeizsäckerWilliams and the dipole gluon distribution. This is a direct consequence of the different gauge-link dependences. By comparing the gluon Sivers functions extracted from DIS and $p p$ collisions, one can test this gauge-link dependence since the Weizsäcker-Williams and dipole-type $T$-odd gluon TMDs are expected to behave differently [16,22].

In DIS, the key to studying the gluon Sivers function is to tag the photon-gluon fusion (PGF) subprocess. It has been shown in Refs. $[25,26]$ that the gluon transverse momentum distribution can be mapped through quark-antiquark jet correlations for the PGF subprocess $\gamma^{*} g \rightarrow q \bar{q}$. The authors of Ref. [22] suggested that the spin asymmetries measured in heavy quark pair and dijet production at an EIC can be used to study the Weizsäcker-Williams (WW) gluon TMDs including the Sivers function. The open charm production in electron-proton scattering $e p^{\uparrow} \rightarrow e^{\prime} c \bar{c} X$ is argued to be an ideal probe to tag the PGF process, and can be investigated at a future EIC. A model study has been carried out in Ref. [2] and the related experimental considerations for tagging charm quark production through $D$ mesons in the final state for the PGF subprocess were discussed in Ref. [27]. In this paper, we will provide detailed information on EIC projections for open charm production with attainable experimental conditions. Alternative methods of tagging the gluon channel through the production of high- $p_{T}$ hadron pairs and dijets are also studied. The advantages and disadvantages of the different channels will be discussed. Table I shows the definitions of the kinematic variables used in this paper.

The remainder of the paper is organized as follows. In Sec. II we discuss the theoretical framework used to build the connection of gluon Sivers function and the size of the single spin asymmetry (SSA). The Monte Carlo setup is described in Sec. III. A detailed description of the results and their projected precision are presented in Sec. IV. We summarize in Sec. V.

TABLE I. Kinematic variables.

\begin{tabular}{|c|c|}
\hline$Q^{2}$ & Virtuality of the exchanged photon \\
\hline$x_{B}$ & Bjorken $x$ \\
\hline$y$ & $\begin{array}{l}\text { Energy fraction of the virtual photon with respect to } \\
\text { the incoming electron }\end{array}$ \\
\hline$W$ & Center-of-mass energy of the $\gamma^{*} p$ system \\
\hline$x$ & $\begin{array}{l}\text { Longitudinal momentum fraction of the quark/gluon } \\
\text { from the polarized proton involved in the hard } \\
\text { interaction }\end{array}$ \\
\hline$z_{h, q}$ & $\begin{array}{l}\text { Energy fraction of a hadron or quark with respect to } \\
\text { virtual photon in the target rest frame }\end{array}$ \\
\hline$k_{\perp}$ & $\begin{array}{l}\text { Initial transverse momentum of gluons inside the } \\
\text { proton in the } \gamma^{*} p \text { center-of-mass frame }\end{array}$ \\
\hline$k_{1 \perp}, k_{2 \perp}$ & $\begin{array}{l}\text { Transverse momentum of the two outgoing partons in } \\
\text { the } \gamma^{*} p \text { center-of-mass frame }\end{array}$ \\
\hline $\begin{array}{l}p_{h 1 \perp} \\
p_{h 2 \perp}\end{array}$ & $\begin{array}{l}\text { Transverse momentum of the trigger/associate } \\
\text { particle in the } \gamma^{*} p \text { center-of-mass frame }\end{array}$ \\
\hline$p_{T}$ & $\begin{array}{l}\text { Transverse momentum of the final-state hadron/jet } \\
\text { with respect to the virtual photon }\end{array}$ \\
\hline$\eta$ & $\begin{array}{l}\text { Pseudorapidity of the final-state hadron/jet in the } \gamma^{*} p \\
\text { center-of-mass frame }\end{array}$ \\
\hline$P_{T}$ & $\begin{array}{l}\text { Transverse momentum scale of the final-state particle/ } \\
\text { jet pair with respect to the virtual photon }\end{array}$ \\
\hline$k_{T}$ & $\begin{array}{l}\text { Vector sum of the transverse momentum for the final- } \\
\text { state hadron/jet pair in the final state }\end{array}$ \\
\hline$\phi_{k S}$ & $\begin{array}{l}\text { Sivers angle, the azimuthal angle difference of } k_{T} \text { and } \\
\text { the proton spin direction }\end{array}$ \\
\hline $\begin{array}{l}p_{\text {TLab }} \\
p_{\text {Lab }}\end{array}$ & $\begin{array}{l}\text { Transverse momentum/momentum of the final-state } \\
\text { hadron in the laboratory frame }\end{array}$ \\
\hline$\eta_{\text {Lab }}$ & $\begin{array}{l}\text { Pseudorapidity of the final-state hadron/jet in the } \\
\text { laboratory frame }\end{array}$ \\
\hline
\end{tabular}




\section{SINGLE SPIN ASYMMETRY ARISING FROM THE GLUON SIVERS EFFECT}

The Sivers function describes the distribution of unpolarized partons with flavor $a$ inside a transversely polarized proton with mass $M_{p}$ and can be expressed following the Trento convention in Ref. [28] as

$\hat{f}_{a / p^{\uparrow}}\left(x, k_{\perp}\right)=f_{a / p}\left(x, k_{\perp}\right)+\frac{1}{2} \Delta^{N} f_{a / p^{\uparrow}}\left(x, k_{\perp}\right) \vec{S} \cdot\left(\hat{\vec{P}} \times \hat{\vec{k}}_{\perp}\right)$.

The first term represents the axially symmetric contribution from the unpolarized parton distribution, while the second term $\Delta^{N} f_{a / p^{\uparrow}}\left(x, k_{\perp}\right)$ generates a distortion away from the center in the number density of unpolarized partons with an intrinsic transverse momentum $\vec{k}_{\perp}$. The azimuthal dependence of this distortion is given by
$\vec{S} \cdot\left(\hat{\vec{P}} \times \hat{\vec{k}}_{\perp}\right)$, where $\vec{P}$ and $\vec{S}$ are the polarized proton three-momentum and spin polarization vector, respectively. The notation $\Delta^{N} f_{a / p^{\uparrow}}\left(x, k_{\perp}\right)$ is related to the Sivers function denoted as $f_{1 T}^{\perp a}\left(x, k_{\perp}\right)$ in the relation $\Delta^{N} f_{a / p^{\uparrow}}\left(x, k_{\perp}\right)=-\frac{2 k_{\perp}}{M_{p}} f_{1 T}^{\perp a}\left(x, k_{\perp}\right)[29]$.

The production of high-transverse-momentum charged hadron pairs or dijets in DIS through $\gamma^{*} g \rightarrow q \bar{q}$ is dominated by gluons, although it may also have some contribution from the quark channel depending on the process measured. The cross section can be calculated in an effective $k_{t}$ factorization framework at leading order as shown in Ref. [30]. If $k_{1}$ and $k_{2}$ are the four-momenta of the outgoing quarks, one can obtain the dihadron cross section as a generalization of the unpolarized case [31] with the transverse momentum imbalance defined as $k_{\perp}=\left|\vec{k}_{1 \perp}+\vec{k}_{2 \perp}\right|$ and the transverse momentum scale as $P_{\perp}=\left|\vec{k}_{1 \perp}-\vec{k}_{2 \perp}\right| / 2$ :

$$
\begin{aligned}
\frac{d \sigma_{\mathrm{tot}}^{\gamma^{*}+p^{\uparrow} \rightarrow h_{1}+h_{2}+X}}{d z_{h 1} d z_{h 2} d^{2} p_{h 1 \perp} d^{2} p_{h 2 \perp}}= & \int_{z_{h 1}}^{1-z_{h 2}} \sum_{q} d z_{q} \frac{z_{q}\left(1-z_{q}\right)}{z_{h 2}^{2} z_{h 1}^{2}} d^{2} p_{1 \perp} d^{2} p_{2 \perp} \hat{f}_{g / p^{\uparrow}}\left(x, k_{\perp}\right) \\
& \times \mathcal{H}_{\mathrm{tot}}^{\gamma^{*} g \rightarrow q \bar{q}}\left(z_{q}, k_{1 \perp}, k_{2 \perp}\right) D_{h 1 / q}\left(\frac{z_{h 1}}{z_{q}}, p_{1 \perp}\right) D_{h 2 / \bar{q}}\left(\frac{z_{h 2}}{1-z_{q}}, p_{2 \perp}\right),
\end{aligned}
$$

where $z_{q}$ is the momentum fraction of the produced quark $q$ with respect to the incoming virtual photon and $\mathcal{H}_{\text {tot }}^{\gamma^{*} g \rightarrow q \bar{q}}$ gives the combined hard factor that incorporates both the longitudinal part $\mathcal{H}^{\gamma_{L}^{*} g \rightarrow q \bar{q}}=\alpha_{s} \alpha_{e m} e_{q}^{2} \frac{8 \hat{s} Q^{2}}{\left(\hat{s}+Q^{2}\right)^{4}}$ and transverse part $\mathcal{H}^{*} g \rightarrow q \bar{q}=\alpha_{s} \alpha_{e m} e_{q}^{2} \frac{\hat{s}^{2}+Q^{4}}{\left(\hat{s}+Q^{2}\right)^{4}}\left(\frac{\hat{u}}{\hat{t}}+\frac{\hat{t}}{\hat{u}}\right)$ of the virtual photon. Equation (2) can be further simplified using the condition $k_{\perp} \ll P_{\perp}$ known as the correlation limit [30]. Equation (2) can thus be expressed as

$$
\begin{aligned}
\frac{d \sigma_{\mathrm{tot}}^{\gamma^{*}+p^{\uparrow} \rightarrow h_{1}+h_{2}+X}}{d z_{h 1} d z_{h 2} d^{2} p_{h 1 \perp} d^{2} p_{h 2 \perp}} & =\int_{z_{h 1}}^{1-z_{h 2}} \sum_{q} d z_{q} \frac{z_{q}^{2}\left(1-z_{q}\right)^{2}}{z_{h 2}^{2} z_{h 1}^{2}} d^{2} p_{1 \perp} d^{2} p_{2 \perp} \alpha_{s} \alpha_{e m} e_{q}^{2} \frac{\left[\left(z_{q}^{2}+\left(1-z_{q}\right)^{2}\right)\left(P_{\perp}^{4}+\epsilon_{f}^{4}\right)+8 z_{q}\left(1-z_{q}\right) P_{\perp}^{2} \epsilon_{f}^{2}\right]}{\left(P_{\perp}^{2}+\epsilon_{f}^{2}\right)^{4}} \\
& \times \hat{f}_{g / p^{\uparrow}}\left(x, k_{\perp}\right) D_{h 1 / q}\left(\frac{z_{h 1}}{z_{q}}, p_{1 \perp}\right) D_{h 2 / \bar{q}}\left(\frac{z_{h 2}}{1-z_{q}}, p_{2 \perp}\right)
\end{aligned}
$$

in which $\epsilon_{f}^{2}$ is related to $Q^{2}$ as $\epsilon_{f}^{2}=z_{q}\left(1-z_{q}\right) Q^{2}$. Choosing the center-of-mass frame of the exchanged virtual photon and the proton, in which the proton beam with momentum $\vec{P}$ is moving in the $-z$ direction, one can obtain an explicit form of the mixed vector product in Eq. (1) as $\vec{S} \cdot\left(\hat{\vec{P}} \times \hat{\vec{k}}_{\perp}\right)=$ $\sin \left(\phi_{k}-\phi_{S}\right)$ with $\phi_{k}$ being the azimuthal angle of $\vec{k}_{\perp}$. A factorized Gaussian parametrization has been adopted for the transverse-momentum-dependent unpolarized parton distribution function $f_{g / p}\left(x, k_{\perp}\right)=f_{g / p}(x) \frac{e^{-k_{\perp}^{2} /\left\langle k_{\perp}^{2}\right\rangle}}{\pi\left\langle k_{\perp}^{2}\right\rangle}$ and fragmentation function $D\left(z, p_{\perp}\right)=D(z) \frac{e^{-p_{\perp}^{2} /\left\langle p_{\perp}^{2}\right\rangle}}{\pi\left\langle p_{\perp}^{2}\right\rangle}$.

There exists a strong correlation between the kinematics of the back-to-back hadron pair and its parent quarks.
Therefore, one can use the variables $P_{T}=\left|\vec{p}_{h 1 \perp}-\vec{p}_{h 2 \perp}\right| / 2$ and $k_{T}=\left|\vec{p}_{h 1 \perp}+\vec{p}_{h 2 \perp}\right|$, which are measurable at the hadron level, to access the underlying parton kinematic variables $P_{\perp}$ and $k_{\perp}$. A schematic illustration of the encoded kinematic variables is shown in Fig. 1. In Sec. IV, we study the precision to which the measurable hadron-level variables represent the parton kinematics. The GSF can be studied in the SSA for dihadron production as follows:

$$
\begin{aligned}
A_{U T}\left(\phi_{k S}, k_{T}\right) & =\frac{d \sigma^{\uparrow}\left(\phi_{k S}, k_{T}\right)-d \sigma^{\downarrow}\left(\phi_{k S}, k_{T}\right)}{d \sigma^{\uparrow}\left(\phi_{k S}, k_{T}\right)+d \sigma^{\downarrow}\left(\phi_{k S}, k_{T}\right)} \\
& \propto \frac{\Delta^{N} f_{g / p^{\uparrow}}\left(x, k_{\perp}\right)}{2 f_{g / p}\left(x, k_{\perp}\right)},
\end{aligned}
$$




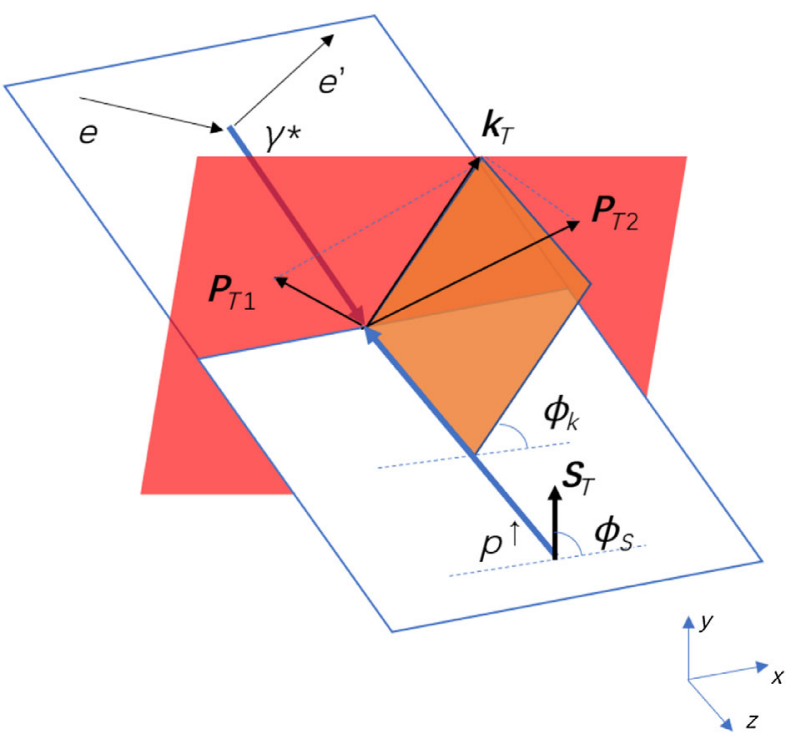

FIG. 1. A schematic illustration of the kinematic variables involved in this measurement.

where the subscript " $U$ " represents the unpolarized electron beam and " $T$ " indicates the transverse polarization of the proton beam. $\phi_{k S}=\phi_{k_{T}}-\phi_{S}$ stands for the angular difference between the total dihadron transverse momentum $\vec{k}_{T}$ and the polarized proton spin direction $\vec{S}_{\perp}$. The amplitude of the SSA is proportional to the corresponding Sivers function divided by the unpolarized parton distributions.

\section{MONTE CARLO SIMULATION SETUP}

In this section, we will describe the setup for our event generation. We use the PYTHIA 6.4 Monte Carlo (MC) program [32] to simulate the unpolarized cross section as expected at an EIC. The PYTHIA generator has been found to reproduce the charged and open charm particle production in the electron-proton collisions at HERA. The comparison of the HERA data $[33,34]$ and the output of the tuned PythiA MC for charged particles and $D^{*}$ mesons is shown in Figs. 2 and 3. Based on this reasonable description of the unpolarized DIS cross section, we will discuss our strategy to obtain the SSA based on weighting the unpolarized results from PYTHIA.

In the simulation, we model the amplitude of the asymmetry as an incoherent superposition of all contributing subprocess on an event-by-event basis. For every event, a weighting factor is obtained according to the kinematics and parton flavor as follows:

$$
w=\frac{\Delta^{N} f_{a / p^{\uparrow}}\left(x, k_{\perp}, Q^{2}\right)}{2 f_{a / p}\left(x, k_{\perp}, Q^{2}\right)} .
$$

At the end, the Monte Carlo asymmetry can be understood as the weighted sum of the asymmetry weights from signal

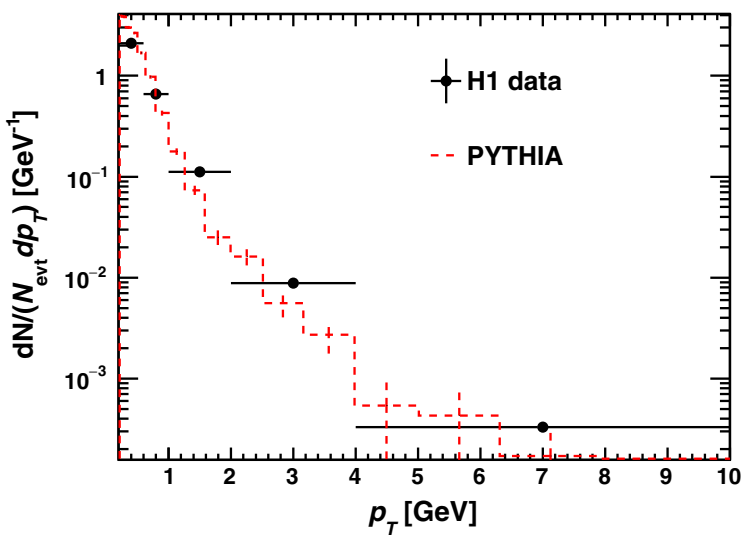

FIG. 2. Charged particle transverse momentum distributions for $0<\eta<1.5$ defined in the virtual photon-hadron center-of-mass frame. The HERA data [33] for $5 \mathrm{GeV}^{2}<Q^{2}<10 \mathrm{GeV}^{2}$, $0.0005<x_{B}<0.002$ with a beam energy $27.6 \mathrm{GeV} \times$ $920 \mathrm{GeV}$ are compared to the tuned PyTHIA results.

(gluon-initiated channels) and background (quark-initiated channels) processes similar to the strategy used in Ref. [35]:

$$
A_{U T}=R_{g} \frac{\Sigma_{i}^{N_{g}} w_{i}}{N_{g}}+R_{q} \frac{\Sigma_{i}^{N_{q}} w_{i}}{N_{q}},
$$

in which $N_{g}$ and $N_{q}$ indicate the number of gluon- and quark-initiated events in the analyzed event sample. The corresponding event fraction is thus obtained as $R_{g}=$ $N_{g} /\left(N_{g}+N_{q}\right)$ and $R_{q}=N_{q} /\left(N_{g}+N_{q}\right)$. In the experiment, it is very hard to reliably separate different subprocesses. Therefore, the fractions of events from different subprocesses are modeled using PYTHIA in this analysis. A validation of this weighting method against experimental

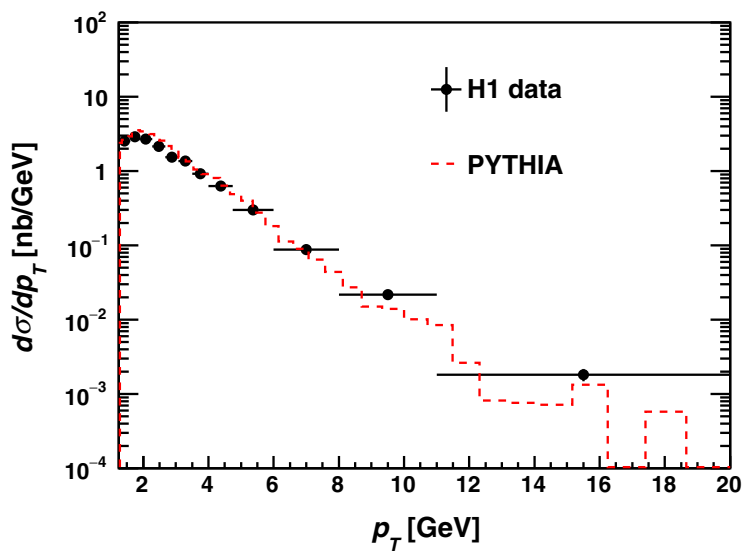

FIG. 3. $\quad D^{*}$ transverse momentum distributions for $\left|\eta_{\text {Lab }}\right|<1.8$ defined in the virtual photon-hadron center-of-mass frame. The HERA data [34] for $5 \mathrm{GeV}^{2}<Q^{2}<100 \mathrm{GeV}^{2}, 0.02<y<0.7$ with the beam energy $27.6 \mathrm{GeV} \times 920 \mathrm{GeV}$ are compared to the tuned PYTHIA results. 


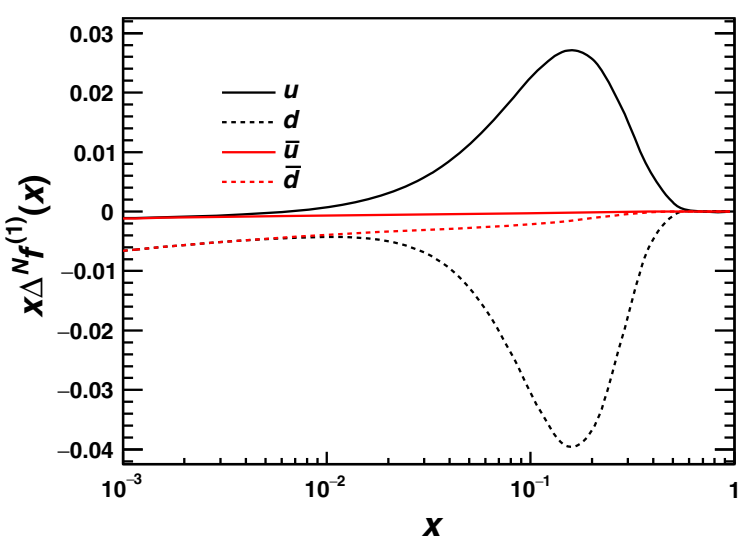

(a)

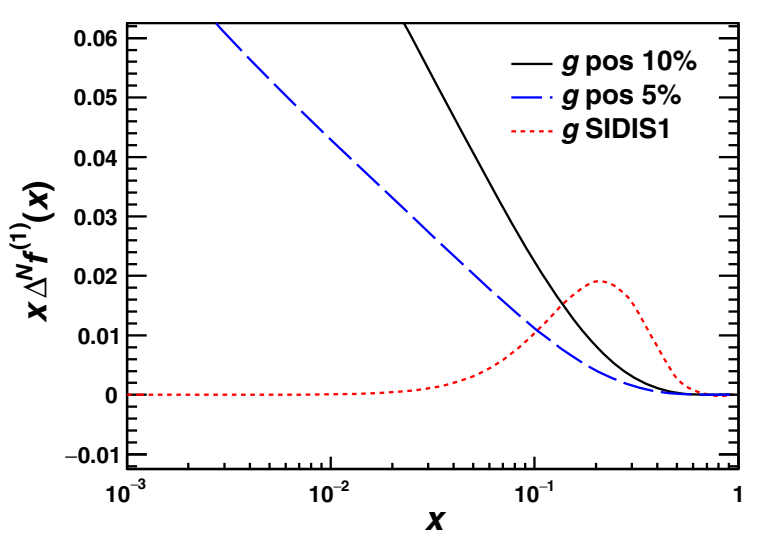

(b)

FIG. 4. The first $k_{\perp}$ moment $x \Delta^{N} f^{(1)}(x)$ of the Sivers function used in this work for quarks (a) and gluons (b) varying with $x$ at the scale $Q^{2}=4 \mathrm{GeV}^{2}$. Sivers moments for $u, d, \bar{u}$ and $\bar{d}$ are displayed by the black solid, black dotted, red solid, and red dotted lines in panel (a). The solid, dashed and dotted lines in panel (b) represent the gluon Sivers function with magnitudes of $10 \%$ and $5 \%$ of the positivity bound, and with the SIDIS fit from Ref. [18], respectively.

data from COMPASS [36] is discussed at the end of this section (see Fig. 5).

The parametrization of the Sivers function is given in a factorized form as

$$
\begin{gathered}
\Delta f_{a / p}=2 \mathcal{N}_{a}\left(x_{a}\right) f_{a / p}\left(x_{a}, Q^{2}\right) h\left(k_{\perp}\right) \frac{e^{-k_{\perp}^{2} /\left\langle k_{\perp}^{2}\right\rangle}}{\pi\left\langle k_{\perp}^{2}\right\rangle}, \\
\mathcal{N}_{a}\left(x_{a}\right)=N_{a} x^{\alpha_{a}}(1-x)^{\beta_{a}} \frac{\left(\alpha_{a}+\beta_{a}\right)^{\left(\alpha_{a}+\beta_{a}\right)}}{\alpha_{a}^{\alpha_{a}} \beta_{a}^{\beta_{a}}}, \\
h\left(k_{\perp}\right)=\sqrt{2 e} \frac{k_{\perp}}{M_{1}} e^{-k_{\perp}^{2} / M_{1}^{2}},
\end{gathered}
$$

in which $f_{a / p}\left(x_{a}, Q^{2}\right)$ is the unpolarized parton distribution, $\mathcal{N}_{a}\left(x_{a}\right)$ and $h\left(k_{\perp}\right) \frac{e^{-k_{\perp}^{2} /\left\langle k_{\perp}^{2}\right\rangle}}{\pi\left\langle k_{\perp}^{2}\right\rangle}$ describe the $x$ and $k_{\perp}$ dependence of the Sivers function. The magnitude of the asymmetry from background contributions is calculated from the quark Sivers function using the recent fits in Ref. [37]:

$$
\begin{aligned}
N_{u_{v}} & =0.18, \quad \alpha_{u_{v}}=1.0, \quad \beta_{u_{v}}=6.2, \\
N_{d_{v}} & =-0.52, \quad \alpha_{d_{v}}=1.9, \quad \beta_{u_{v}}=10.0, \\
N_{\bar{u}} & =-0.01, \quad N_{\bar{d}}=-0.06, \quad M_{1}^{2}=0.8 \mathrm{GeV}^{2} .
\end{aligned}
$$

For the gluon Sivers function we utilize two models as input to our study. The first model is the SIDIS1 set obtained in the fit in Ref. [18], which follows a similar parametrization form as the quark Sivers function with the parameters given by

$N_{g}=0.65, \quad \alpha_{g}=2.8, \quad \beta_{g}=2.8, \quad M_{g}^{2}=0.43 \mathrm{GeV}^{2}$.

The second gluon Sivers model relies on the positivity bound assumption used in Ref. [38]:

$$
f_{1 T}^{\perp g}=-\frac{2 \sigma M_{p}}{k_{\perp}^{2}+\sigma^{2}} f_{g}\left(x, k_{\perp}\right), \quad \sigma=0.8 \mathrm{GeV}
$$

in which the positivity limit is saturated when $k_{\perp}=$ $0.8 \mathrm{GeV}$. We will use $10 \%$ and $5 \%$ of the positivity bound to study quantitatively the measurability of the gluon Sivers function. We calculate the weight of every event according to the inputs discussed here to obtain the magnitude of the asymmetry in the final state. An example of the first $k_{\perp}$ moment of the input Sivers distribution $\Delta^{N} f^{(1)}(x)=\int d^{2} k_{\perp} \frac{k_{\perp}}{4 m_{p}} \Delta^{N} f_{a / p^{\uparrow}}\left(x, k_{\perp}\right)$ is shown in Fig. 4. Figure 4(a) shows the quark Sivers functions used to estimate the background contribution, while the gluon Sivers functions are shown in Fig. 4(b). For the current parametrizations the quark Sivers functions have a maximum for $x>0.1$ for the valence quarks and become negligible in the small- $x$ regime. The magnitude of the sea-quark Sivers functions is small over the entire $x$ range. It is noticeable that the gluon Sivers functions based on the positivity bound assumption and SIDIS1 set have quite different functional forms in $x$.

We provide in Fig. 5(a) a comparison of the charged hadron asymmetry measured by the COMPASS experiment [36] with the asymmetry obtained from weighting PYTHIA events according to the method described above with the quark Sivers functions. It is not surprising to see that radiation effects modeled by the parton shower mechanism in Pythia are quite weak at the COMPASS energy. The comparison also shows that one can describe both positive and negative charged hadron asymmetries from COMPASS with the event weighting method.

It should be explicitly noted that the parametrizations of the Sivers asymmetry discussed here are not accounting for 


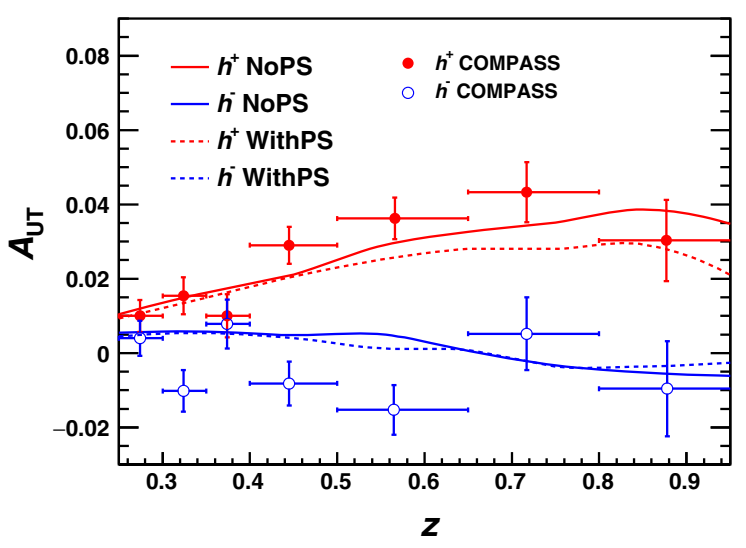

FIG. 5. Comparison of the charged hadron asymmetry measured by COMPASS with the one from the weighting method. The COMPASS data are taken from Ref. [36]. Radiation effects are estimated by turning the parton shower mechanism on (WithPS) and off (NoPS) in PYTHIA, shown with dotted and solid lines in this comparison.

any effects due to the QCD scale dependence of TMDs. The QCD evolution of the Sivers function can be calculated in the QCD resummation formalism following the CollinsSoper-Sterman method $[39,40]$ by applying the correct Sudakov factor to the spin-dependent parton distributions [41-43]. The necessary precise phenomenological inputs to determine the QCD scale evolution of the Sivers asymmetry are not yet available, but they can be obtained from the future RHIC and EIC measurements. We will therefore not address the evolution of the gluon Sivers function in this paper but leave it for future work.

In order to estimate the statistical uncertainty of the SSA in our simulation, we use $\delta A=\sqrt{\frac{1}{P^{2} N}-\frac{A^{2}}{N}}$ from Ref. [44], where $N$ represents the count of selected pairs in a certain kinematic bin, and $P$ indicates the polarization of the proton beam. In this work, we assume a polarization $P=70 \%$ for the EIC beam energy configuration of $20 \mathrm{GeV} \times 250 \mathrm{GeV}$ with an integrated luminosity $\mathcal{L}_{\text {int }}=10 \mathrm{fb}^{-1}$.

\section{RESULTS AND DISCUSSIONS}

In this study, the event kinematics has been restricted to $0.01<y<0.95$ and $1 \mathrm{GeV}^{2}<Q^{2}<20 \mathrm{GeV}^{2}$ with the electron and proton beam energy configuration of $20 \mathrm{GeV} \times 250 \mathrm{GeV}$. A detector system specially designed for EIC with a wide acceptance $-4.5<\eta_{\text {Lab }}<4.5$ for measuring charged particles [45] has been assumed, in which case the event kinematics can be well reconstructed from the scattered electron. This selection gives an average event kinematics for the minimum bias events as $\left\langle x_{B}\right\rangle=0.0012,\left\langle Q^{2}\right\rangle=2.5 \mathrm{GeV}^{2},\langle W\rangle=54.6 \mathrm{GeV}$. The wide kinematic reach at this high center-of-mass energy $(\sqrt{s}=141 \mathrm{GeV})$ makes it possible to study the evolution of the Sivers asymmetry and to access the region dominated by gluons.
As discussed in Sec. II, there is a correlation between the vector sum of the transverse momentum $k_{T}$ for the selected hadron pairs or dijets with the initial transverse motion of gluons. It then follows naturally to investigate the Sivers asymmetry through the sine modulation for the angle $\phi_{k S}=\phi_{k_{T}}-\phi_{S}$, which defines the difference between $k_{T}$ and the spin direction of the proton. To tag the gluon distributions and study the Sivers asymmetries we will study the $D$ meson pair, charged dihadrons and dijet production. The detailed experimental cuts for each channel are listed as follows:

(1) $D$ meson pair production:

$$
\begin{gathered}
D^{0} \rightarrow \pi K, \quad\left|\eta_{\mathrm{Lab}}^{\pi / K}\right|<3.5, \quad p_{T \mathrm{Lab}}^{\pi / K}>0.2 \mathrm{GeV}, \\
p_{T}^{D}>0.7 \mathrm{GeV}, z_{D}>0.1 .
\end{gathered}
$$

(2) Charged dihadron production:

$$
-4.5<\eta_{\mathrm{Lab}}<4.5, p_{T}^{h}>1.4 \mathrm{GeV}, z_{h}>0.1 \text {. }
$$

(3) Dijet production:

$\pi, K, p, \gamma$ with $p_{T \text { Lab }}>0.25 \mathrm{GeV},\left|\eta_{\text {Lab }}\right|<4.5$ used for the jet reconstruction with the anti- $k_{T}$ algorithm and a cone radius $R=1$; the trigger jet has $p_{T}^{\text {jet } 1}>4.5 \mathrm{GeV}$ and the associated jet has $p_{T}^{\mathrm{jet} 2}>4 \mathrm{GeV}$.

\section{A. The gluon SSA in open charm production}

Heavy flavor production has been proven to be very useful for measuring the gluon Sivers asymmetry in protonproton collisions as shown in Refs. [38,46]. Similar to the case in hadron-hadron reactions, it is well accepted that heavy-flavor production in DIS is a very clean channel to directly probe the gluon distributions. In this section we demonstrate the possibility to measure the gluon Sivers function in open charm production $\gamma^{*} g \rightarrow c \bar{c}$ with $D^{0}$ mesons in the final state. Open charm production has the advantage that quark-initiated processes are strongly suppressed and one becomes essentially only sensitive to gluon-initiated subprocesses. The $D^{0}$ mesons are identified through the $\pi K$ decay channel by taking advantage of the vertex tracking detector integrated into the main detector. The momentum and pseudorapidity distribution of the $K$ meson from the $D^{0}$ decay can be found in Fig. 6. The $K$ momenta are typically a few $\mathrm{GeV}$ in the central rapidity region, and extend to $10 \mathrm{GeV}$ at rapidities $|\eta|>1$. The distribution for the $\pi$ mesons from the $D^{0}$ decay is found to be very similar to the one from $K$ mesons. The $D^{0}$ meson decay products are required to be in $\left|\eta_{\text {Lab }}^{\pi / K}\right|<3.5$ and to have transverse momenta $p_{T \mathrm{Lab}}^{\pi / K}>0.2 \mathrm{GeV}$ to be reconstructed and identified. The $p_{T \mathrm{Lab}}$ correlation of the $D^{0}$ meson decay products is shown in Fig. 7; most of the $\pi, K$ products pass the transverse momentum cut. The kinematics of a directly produced $D^{0}$ meson and for one from the decay of a heavier charm meson are basically the same, and therefore all $D^{0}$ mesons with $p_{T}>0.7 \mathrm{GeV}$ and $z_{h}>0.1$ are included in this study. 


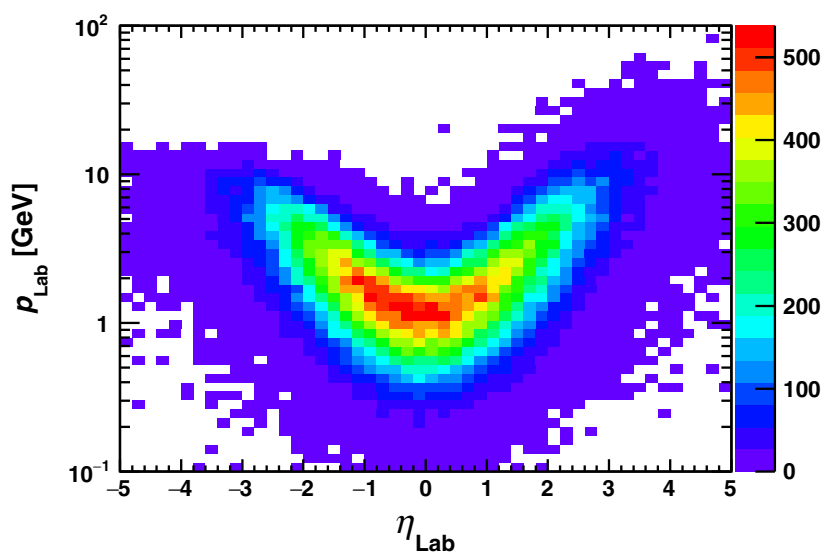

FIG. 6. Momentum-pseudorapidity correlation for $K$ decayed from a $D^{0}$ meson.

To capture the full charm-anticharm quark pair kinematics, we select events with $D \bar{D}$ pairs in the final state. Figure 8 shows that gluon-initiated processes account for about $90 \%$ of the total selected events over a wide range in $x_{B}$ for two $Q^{2}$ bins. For $x_{B}>0.1$ quark-initiated subprocesses become slightly more important.

The sensitivity of the $D \bar{D}$ pair measurement to the magnitude of the gluon Sivers function is shown in Fig. 9(a). The statistical uncertainty is based on an integrated luminosity of $\mathcal{L}_{\text {int }}=10 \mathrm{fb}^{-1}$. The solid curve represents the parton-level asymmetry. The Sivers asymmetry based on the scenario with $10 \%$ of the positivity bound of the gluon Sivers function is indicated by the black filled symbols. The SSA for the background quark-initiated Sivers effect is consistent with zero and not shown here. The limited statistical precision for the $D \bar{D}$ final state due to the small branching ratio $(3.87 \% D \rightarrow K \pi)$ makes it challenging to precisely determine the gluon Sivers function at the level of $10 \%$ of the positivity bound. Therefore, we also investigated the sensitivity to the magnitude of the gluon Sivers function requiring only one $D$ meson. The

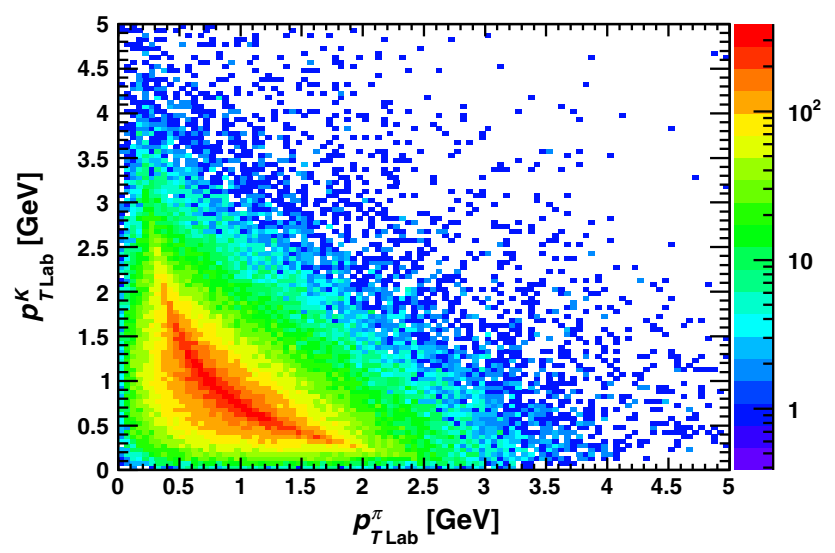

FIG. 7. $p_{T}$ correlation for $\pi$ and $K$ decayed from the same $D$ meson.

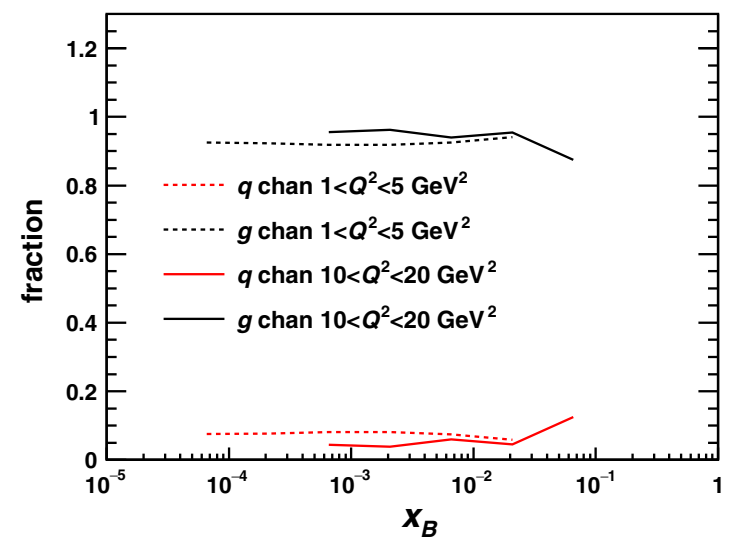

FIG. 8. Fraction of underlying subprocesses initiated by quarks (red curves) or gluons (black curves) for $D \bar{D}$ pair production. The solid and dotted curves represent the two $Q^{2}$ bins of $10<Q^{2}<$ $20 \mathrm{GeV}^{2}$ and $1<Q^{2}<5 \mathrm{GeV}^{2}$.

Sivers angle $\phi_{k S}$ is calculated by replacing $k_{T}$ with the $D$ meson transverse momentum. Figure 9(b) depicts the SSA based on the assumption that the gluon Sivers function has a magnitude of $10 \%$ of the positivity bound, which can be well distinguished from the background SSA due to quark Sivers effects. Comparing Figs. 9(b) and 9(a), one can observe that the initial parton-level asymmetries are similar, but the magnitude of the final-state asymmetry for single $D$ mesons is reduced since the transverse momentum of one $D$ meson is not a good proxy for the initial gluon transverse momentum.

A similar approach to open charm production is to select $K^{+} K^{-}$pairs in the final state, which enhances the underlying process $\gamma^{*} g \rightarrow s \bar{s}$. We find in our study that this measurement is also statistically limited and can only resolve a gluon Sivers signal up to $10 \%$ of the positivity bound. Since the global features of this measurement are similar to the dihadron case, which will be discussed in the next section, we will not provide more information.

\section{B. Gluon SSA through charged dihadron pairs}

In SIDIS production, the leading-order DIS (LODIS) process $\gamma^{*} q \rightarrow q$ accounts for a large fraction of the charged particle production. The LODIS process can be largely suppressed by requiring a pair of high- $p_{T}$ charged hadrons.

The acceptance for the charged hadrons is required to fit the EIC detector design $\left|\eta_{\text {Lab }}\right|<4.5$ and the hadron pair candidates must have a transverse momentum $p_{T}^{h}>$ $1.4 \mathrm{GeV}$ and $z_{h}>0.1$. To select hadron pairs from back-toback jets, we ask that $k_{T}<0.7 P_{T}$ with $P_{T}=\left|\vec{p}_{T}^{h 1}-\vec{p}_{T}^{h 2}\right| / 2$. This way, one can eliminate the contribution of two hadrons fragmented from the same parton. The event fractions affected by the gluon- and quark-initiated processes are shown in Fig. 10. Around $80 \%$ of the high- $p_{T}$ dihadron events are generated from gluon-initiated 


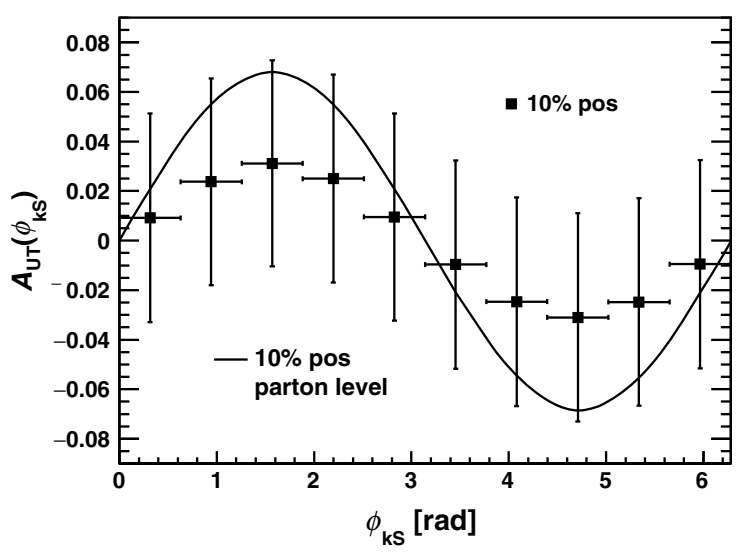

(a)

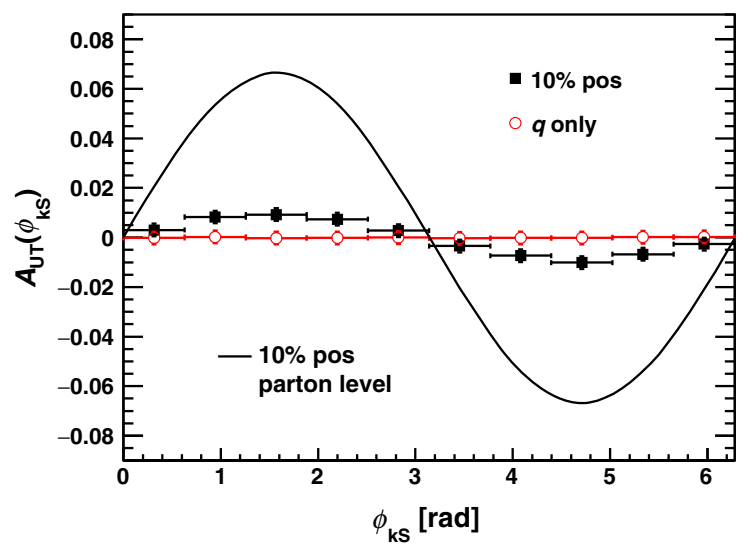

(b)

FIG. 9. Projection for the SSA dependence on $\phi_{k s}$ shown for different inputs for the gluon Sivers function for $D \bar{D}$ pairs (a) and single $D$ mesons (b). The vertical bars represent the statistical uncertainties obtained with the kinematic cuts $\left|\eta_{\text {Lab }}^{\pi / K}\right|<3.5$, $p_{\text {TLab }}^{\pi / K}>0.2 \mathrm{GeV}, z^{D}>0.1, p_{T}^{D}>0.7 \mathrm{GeV}, 0.01<y<0.95$ and $1 \mathrm{GeV}^{2}<Q^{2}<20 \mathrm{GeV}^{2}$ at the electron-proton beam energy $20 \mathrm{GeV} \times 250 \mathrm{GeV}$ and an integrated luminosity $\mathcal{L}_{\text {int }}=10 \mathrm{fb}^{-1}$. The initial parton-level asymmetry is shown with the solid line. The Sivers asymmetry for $10 \%$ of the positivity bound and for the quark contribution are displayed with the closed squares and the open circles, respectively.

processes in the small- $x_{B}$ region. The fraction of quarkinitiated processes grows rapidly as $x_{B}$ approaches 0.1 and with increasing $Q^{2}$. This behavior with $Q^{2}$ is due to the fact that more high- $p_{T}$ hadrons are generated through QCD radiation, which has an increased probability with increasing $Q^{2}$.

In particular, we note that an understanding of the gluon Sivers function requires measuring its dependence on $x_{B}$ and $Q^{2}$. Figure 11 compares the SSA for charged hadron pairs assuming that the magnitude of the gluon Sivers function is $5 \%$ of its positivity bound (solid circles) and the SIDIS1 set (solid triangles) as well as no gluon Sivers contribution, but a quark Sivers contribution (open circles).

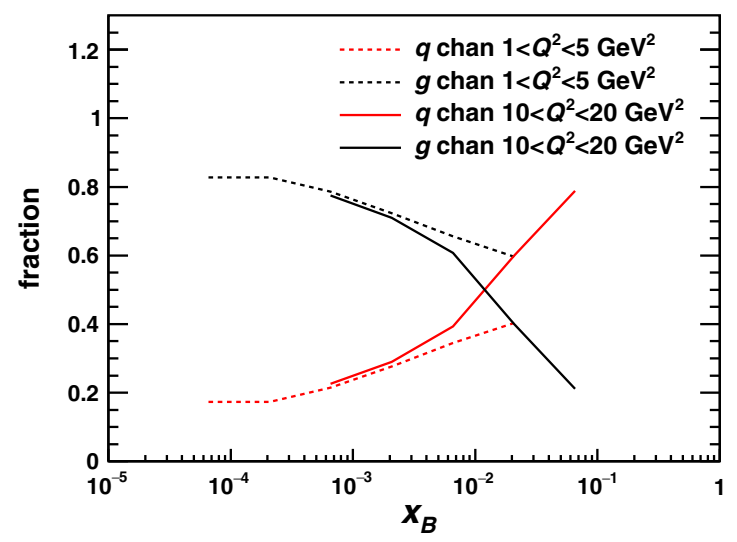

FIG. 10. Fraction of the underlying subprocesses initiated by quarks (red curves) or gluons (black curves) for high- $p_{T}$ charged dihadron pairs. The solid and dotted curves represent the $Q^{2}$ bins of $10<Q^{2}<20 \mathrm{GeV}^{2}$ and $1<Q^{2}<5 \mathrm{GeV}^{2}$, respectively.

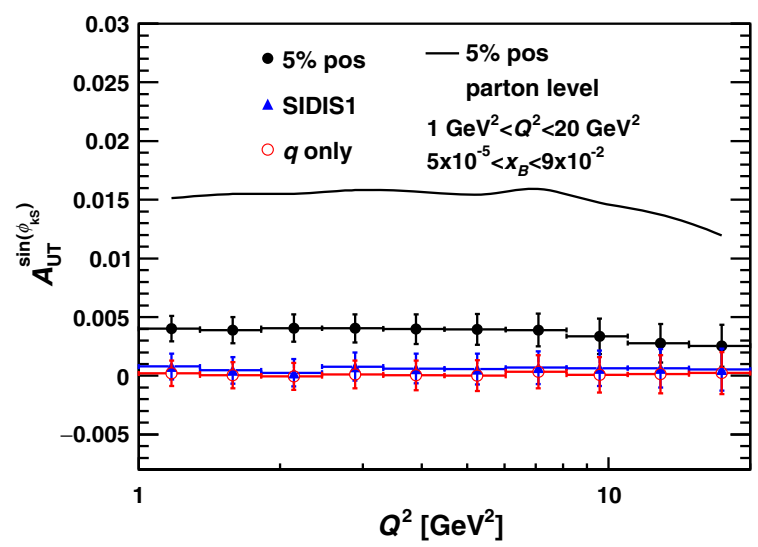

(a)

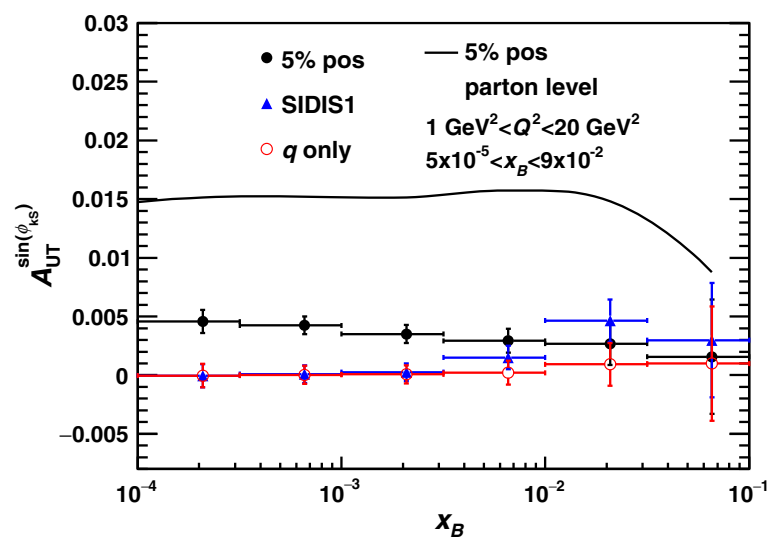

(b)

FIG. 11. Sivers asymmetries dependent on $Q^{2}$ (a) and $x_{B}$ (b) for charged hadron pairs requiring the cuts $\left|\eta_{\text {Lab }}^{h}\right|<4.5, p_{T}^{h}>$ $1.4 \mathrm{GeV}, z^{h}>0.1, k_{T}<0.7 P_{T}, 0.01<y<0.95$ and $1 \mathrm{GeV}^{2}<$ $Q^{2}<20 \mathrm{GeV}^{2}$ for the electron-proton beam energy $20 \mathrm{GeV} \times$ $250 \mathrm{GeV}$ for an integrated luminosity $\mathcal{L}_{\text {int }}=10 \mathrm{fb}^{-1}$. 
The study shows that a gluon Sivers function with a magnitude of $5 \%$ of the positivity bound can be measured at an EIC. We also find that the initial parton-level asymmetry is significantly diluted (by a factor of 3 ), as can be seen by comparing the black curve and the solid circles. This dilution is larger than the one shown in Fig. 9(a) for $D \bar{D}$ pairs (a factor of 2). The increase can be explained by the stronger smearing (due to the fragmentation of light quarks to hadrons) of the correlation between the parton and the hadron $p_{T}$.

Figure 11 shows that the angular modulation of the Sivers function is only weakly dependent on $Q^{2}$, because of the missing TMD evolution in the current framework, but it is much more sensitive to $x_{B}$. The dependence on $x_{B}$ is a natural consequence of the behavior of the Sivers function parametrization with $x$.

\section{Gluon SSA in dijet production}

Comparing the hadron-level observables with jets, it can be clearly seen that jets provide a more precise reconstruction of the initial gluon kinematics. In the following we study the sensitivity to the gluon Sivers function in dijet production. The jets are reconstructed from charged hadrons ( $\pi, K$ and protons) measured in the central tracker together with photons accepted in the calorimeter requiring a minimum transverse momentum $p_{\text {TLab }}>$ $0.25 \mathrm{GeV}$ and $\left|\eta_{\text {Lab }}\right|<4.5$. The jet radius parameter is assumed to be $R=1$ in the anti- $k_{T}$ jet reconstruction algorithm. Dijet events are defined with the trigger jet $p_{T}^{\text {jet1 }}>4.5 \mathrm{GeV}$ and the associated jet $p_{T}^{\text {jet2 }}>4 \mathrm{GeV}$. Similar to the dihadron channel, we use the vector sum of the transverse momenta of the two jets $k_{T}=\mid \vec{p}_{T}^{\text {jetl }}+$ $\vec{p}_{T}^{\mathrm{jet} 2} \mid$ as the proxy to access the underlying gluon dynamics. We present the fractions for quark- and gluon-initiated processes in Fig. 12. The quark fraction is substantial (close to $30 \%$ ) for low- $Q^{2}$ events even for $x_{B} \sim 10^{-4}$. The fraction

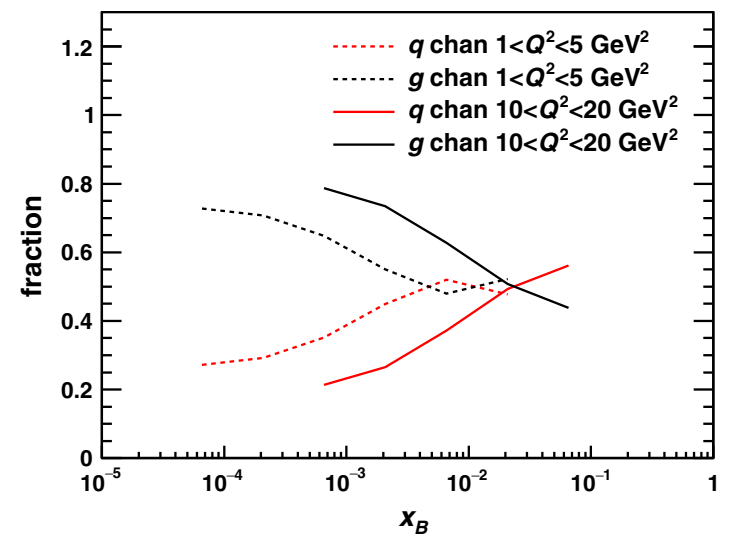

FIG. 12. Underlying subprocess fraction initiated by quarks (red curves) or gluons (black curves) for dijet production. The solid and dotted curves represent the $Q^{2}$ bins of $10<Q^{2}<$ $20 \mathrm{GeV}^{2}$ and $1<Q^{2}<5 \mathrm{GeV}^{2}$, respectively. of gluon-initiated channels is maximized at small $x_{B}$ and drops below the quark fraction if $x_{B}$ is close to 0.01 or 0.1 depending on the $Q^{2}$ range. Unlike for the dihadron case, the gluon event fraction increases with $Q^{2}$.

In Fig. 13, it is observed that a gluon Sivers function with a magnitude of $5 \%$ of the positivity bound or the SIDIS1 set can be well separated from a SSA based on the quark Sivers effect at large $x_{B}$ for an integrated luminosity of $\mathcal{L}_{\text {int }}=10 \mathrm{fb}^{-1}$. Despite the fact that the initial parton asymmetry for the dijet process is smaller than that for the dihadron channel, a larger fraction of the initial asymmetry survives in the dijet channel. The shape of the initial parton-level asymmetry is largely preserved in the dijet asymmetry in all kinematic variables. This is very helpful for exploring the dependence of the gluon Sivers function on the hard-scattering kinematics. Due to the strong correlation between the momentum of a jet and its mother parton, it is possible to reconstruct the momentum fraction of the parton participating in the hard interaction from the dijet momentum information: $x_{\text {parton }}^{\text {rec }}=$ $\left(p_{T}^{\mathrm{jet} 1} e^{-\eta^{\mathrm{jet} 1}}+p_{T}^{\mathrm{jet} 2} e^{-\eta^{\mathrm{je} 2 \mathrm{2}}}\right) / W$. The SSA as a function of $x_{\text {parton }}^{r e c}$ is shown in Fig. 13(c). The initial functional form of the gluon Sivers function in $x$ is well reproduced in the measured SSA as a function of $x_{\text {parton. }}^{\text {rec }}$. The SSA based on a gluon Sivers function with a magnitude of $5 \%$ of the positivity bound decreases while the one based on the SIDIS1 set increases with $x_{\text {parton }}^{\text {rec }}$. The shape of the initial gluon Sivers function is largely the same in the measured SSA. This will allow to distinguish different gluon Sivers models.

With projected high statistics for the dijet channel, the evolution of the GSF with $Q^{2}$ and $x_{B}$ can be studied utilizing a multidimensional binning. Figure 14 shows the SSA based on the gluon Sivers function from the SIDIS1 set as a function of $Q^{2}$ in three $x_{B}$ bins. One of the differential features of the SIDIS1 gluon Sivers function is that the asymmetry increases in the high- $x_{B}$ bins, which is consistent with the behavior in $x_{B}$. The decrease of the SSA as a function of $Q^{2}$ is seen especially in the high- $x_{B}$ bins. This signature can be utilized to study the evolution of the gluon Sivers function in the dijet channel.

A more detailed analysis on the main cause of the smearing from the parton-level asymmetry to the measured asymmetry in dijet production is shown in Fig. 15. We present a comparison of the observed Sivers asymmetry in the dijet measurement with different hadronization assumptions to the probed parton-level asymmetry. To study the influence of $p_{T}$ in the hadronization as well as the effect due to the decay of particle resonances, we have turned both processes consecutively off in the simulation by setting the respective PYTHIA parameters [PARJ(21) and $\operatorname{MSTJ}(21)]$ to zero. The solid red and black curves in Fig. 15 represent the parton-level and measured asymmetry, respectively. Comparing the dotted curve (fragmentation 


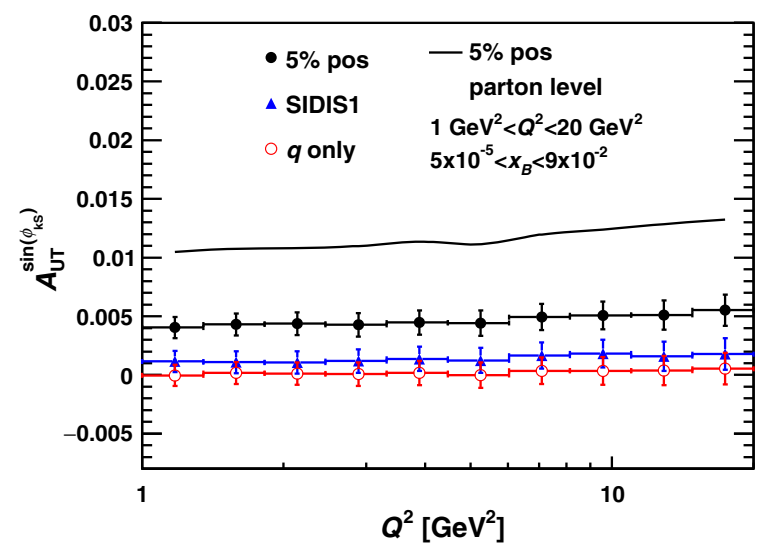

(a)

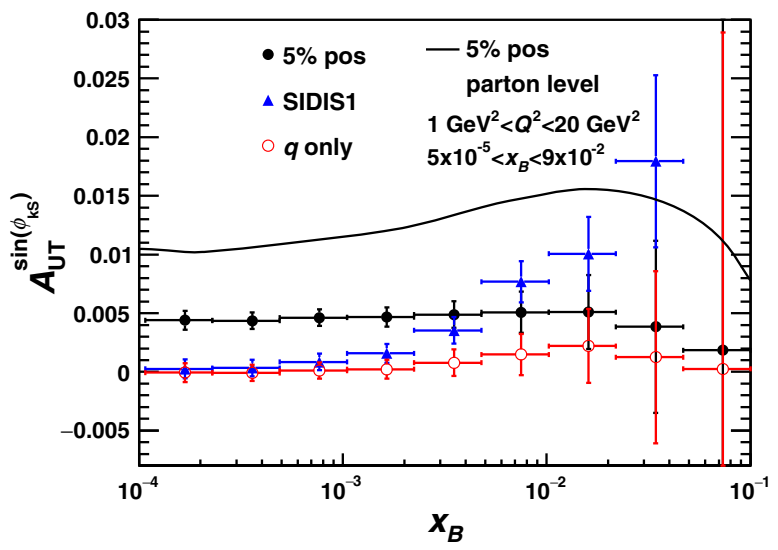

(b)

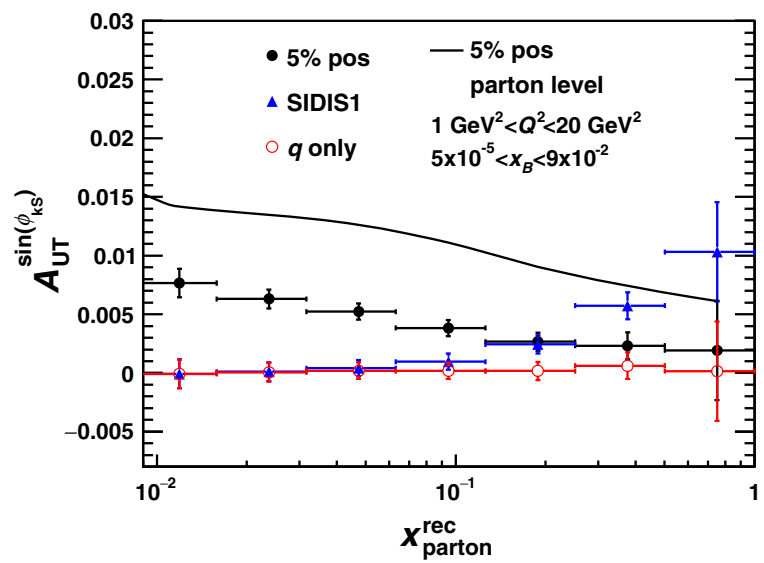

(c)

FIG. 13. SSA modulation dependent on $Q^{2}$ (a), $x_{B}$ (b) and $x_{\text {parton }}^{\text {rec }}(\mathrm{c})$ for the dijet channel with the following kinematic cuts: trigger jet $p_{T}^{\text {jet1 }}>4.5 \mathrm{GeV}$ and associated jet $p_{T}^{\text {jet2 }}>4 \mathrm{GeV}$, $0.01<y<0.95$ and $1 \mathrm{GeV}^{2}<Q^{2}<20 \mathrm{GeV}^{2}$ at the electronproton beam energy $20 \mathrm{GeV} \times 250 \mathrm{GeV}$ with an integrated luminosity $\mathcal{L}_{\text {int }}=10 \mathrm{fb}^{-1}$.

$p_{T}$ off) and the dashed curve (particle resonance decay and fragmentation $p_{T}$ off) shows clearly that the dominant effect is due to resonance decay in the fragmentation. As shown in Fig. 15(b) (the asymmetry varying with $x_{B}$ ), we

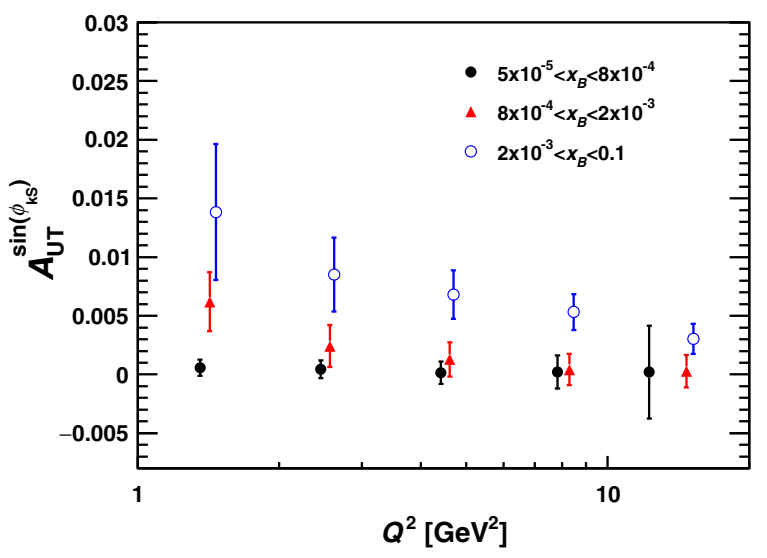

FIG. 14. Sivers modulation for the dijet channel as a function of $Q^{2}$ for three $x_{B}$ bins using the SIDIS1 gluon Sivers function as input.

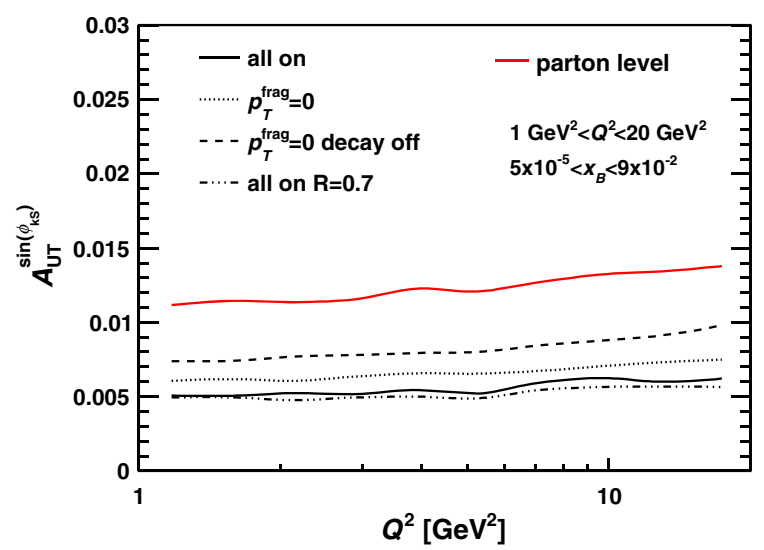

(a)

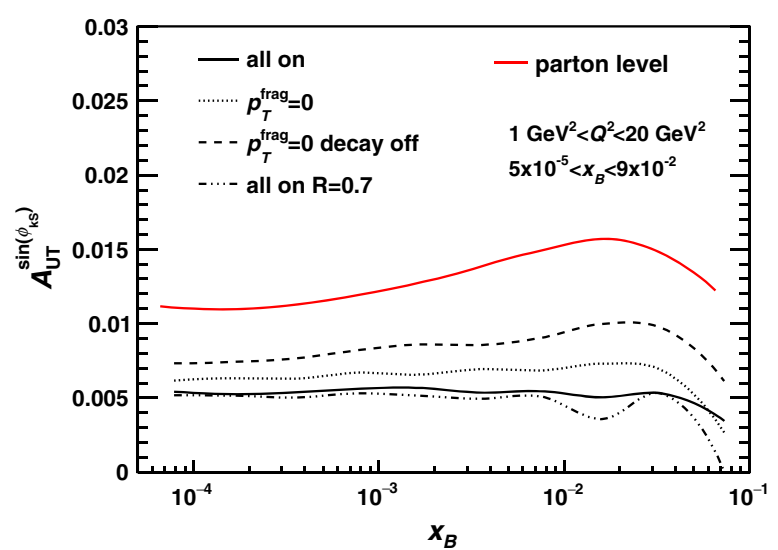

(b)

FIG. 15. Sivers asymmetries for dijets for different hadronization assumptions. Shown are the initial parton-level asymmetry (red solid curve) and the dijet asymmetry (black solid curve). The dotted curve represents the dijet Sivers SSA without fragmentation $p_{T}$, while the dashed curve represents the dijet Sivers SSA without fragmentation $p_{T}$ and with the resonance decays in the fragmentation turned off. The dash-dotted line represents the dijet asymmetry with a cone size of $R=0.7$. 


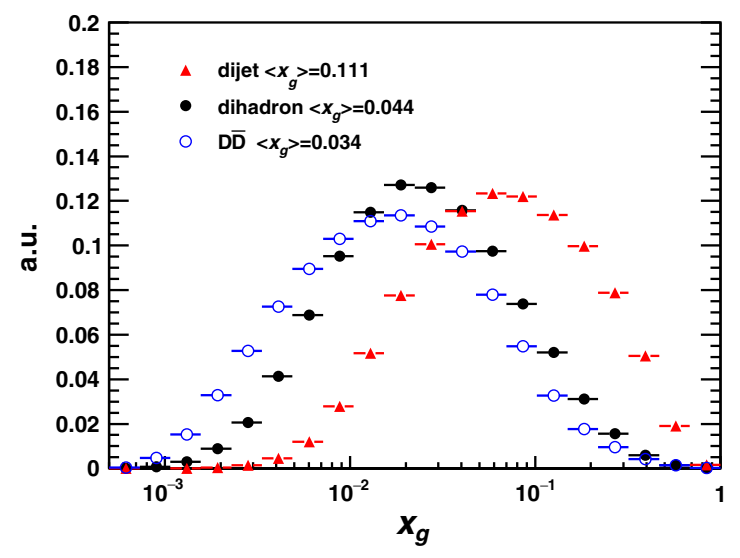

FIG. 16. $x_{g}$ coverage probed by all the different observables.

observe that the resonance decay becomes slightly more important in the high- $x_{B}$ region. The remaining dilution of the parton-level asymmetry is caused by QCD radiation, the $p_{T}$ dependence of the hard-scattering process and the ability to measure a small transverse momentum imbalance with high- $p_{T}$ dijets. We also perform a study on the impact of using different algorithms and cone sizes in the jet reconstruction procedure. It is found that the effect of changing the jet reconstruction algorithm from anti- $k_{T}$ to $k_{T}$ or the cone size from $R=1$ to $R=0.7$ is barely visible. We present the result for the dijet asymmetry with a cone size $R=0.7$ with the dash-dotted line in Fig. 15. The choice of a smaller cone size only leads to a slightly smaller asymmetry. It is implied in this comparison that the dijet asymmetry size is rather robust in spite of the jet reconstruction methods.

An important aspect in comparing the different channels is the coverage in the gluon momentum fraction $x_{g}$. Figure 16 shows that the $x_{g}$ distributions for the different channels are complementary. Both the heavy flavor $D \bar{D}$ mesons and the dihadrons probe lower values of $x_{g}\left(\left\langle x_{g}\right\rangle \sim 0.03\right)$ and the dijet channel probes the larger $x_{g}$ range $\left(\left\langle x_{g}\right\rangle \sim 0.1\right)$.

\section{SUMMARY}

We have performed a study on the feasibility of measuring the gluon Sivers function in high- $p_{T}$ charged dihadrons, heavy-flavor mesons and dijet production in polarized $e p^{\uparrow}$ collisions at an EIC. Scanning different assumptions of the magnitude of the gluon Sivers function provides a systematic study on the sensitivity of the different channels.

We found that although the heavy-flavor $D \bar{D}$ meson production is the cleanest channel to tag gluon-initiated processes, it is at the same time also the most statistically challenging process and therefore the sensitivity to small gluon Sivers effects is limited. An alternative method using inclusive $D$ mesons provides sensitivity to a gluon Sivers function with a magnitude of $10 \%$ of the positivity bound for a nominal integrated luminosity of $\mathcal{L}_{\text {int }}=10 \mathrm{fb}^{-1}$. But the smearing between the parton-level and measured asymmetries is significantly increased. The high- $p_{T}$ charged dihadron channel is statistically more favorable and can resolve a magnitude of the gluon Sivers function down to $5 \%$ of the positivity bound. The most precise analyzer for the gluon Sivers effects at an EIC is the dijet channel; due to its statistical advantage it provides the best sensitivity even for the small Sivers effects and can span the largest $Q^{2}$ range to study TMD evolution effects. Due to its tight correlation between parton and jet kinematics, it has the smallest dilution between the parton-level and measured asymmetries. Overall it is thus the most promising experimental channel to determine and study all features of the gluon Sivers effect at the future EIC.

Following the classification of the unpolarized gluon TMDs, the gluon Sivers function can also be separated into WW-type and dipole-type TMDs depending on the gaugelink structure involved in the process. Quark-antiquark production in DIS probes the WW gluon Sivers function and is related to the one measured in photon pair production in proton-proton collisions through a sign flip as discussed in Sec. I. We note that the dipole-type gluon Sivers function can only be accessed through the $p^{\uparrow} p \rightarrow \gamma$ jet $X$ process. The future EIC project will play an important role in providing complementary information to further our understanding of the different types of gluon TMDs, in particular for the gluon Sivers function by testing the predicted sign flip of the WW gluon Sivers function if measured in $e p$ and $p p$ collisions.

\section{ACKNOWLEDGMENTS}

We would like to thank M. Diehl and Zhong-Bo Kang for helpful suggestions and discussions. We are grateful to A. Prokudin for providing us with their recent parametrizations for the quark Sivers function. E. C. A. and J. H. L. acknowledge the support by the U.S. Department of Energy under Contract No. DE-SC0012704. This work was supported by the Fundamental Research Funds for the Central Universities, China University of Geosciences (Wuhan) No. CUG180615, the National Key Research and Development Program of China (2016YFE0100900) and the NSFC (No. 11475068, No. 11575070). 
[1] M. G. Perdekamp and F. Yuan, Annu. Rev. Nucl. Part. Sci. 65, 429 (2015).

[2] D. Boer et al., arXiv:1108.1713.

[3] D. W. Sivers, Phys. Rev. D 41, 83 (1990).

[4] A. V. Efremov, K. Goeke, S. Menzel, A. Metz, and P. Schweitzer, Phys. Lett. B 612, 233 (2005).

[5] J. C. Collins, Phys. Lett. B 536, 43 (2002).

[6] A. Airapetian et al. (HERMES Collaboration), Phys. Rev. Lett. 94, 012002 (2005).

[7] M. Alekseev et al. (COMPASS Collaboration), Phys. Lett. B 673, 127 (2009).

[8] X. Qian et al. (Jefferson Lab Hall A Collaboration), Phys. Rev. Lett. 107, 072003 (2011).

[9] M. Anselmino, M. Boglione, U. D’Alesio, A. Kotzinian, S. Melis, F. Murgia, A. Prokudin, and C. Turk, Eur. Phys. J. A 39, 89 (2009).

[10] L. Adamczyk et al. (STAR Collaboration), Phys. Rev. D 86, 051101 (2012).

[11] A. Adare et al. (PHENIX Collaboration), Phys. Rev. D 82, 112008 (2010); 86, 099904(E) (2012).

[12] L. Adamczyk et al. (STAR Collaboration), Phys. Rev. Lett. 116, 132301 (2016).

[13] M. Aghasyan et al. (COMPASS Collaboration), Phys. Rev. Lett. 119, 112002 (2017).

[14] A. Accardi et al., Eur. Phys. J. A 52, 268 (2016).

[15] H. H. Matevosyan, A. Kotzinian, E.-C. Aschenauer, H. Avakian, and A. W. Thomas, Phys. Rev. D 92, 054028 (2015).

[16] D. Boer, C. Lorc, C. Pisano, and J. Zhou, Adv. High Energy Phys. 2015, 371396 (2015).

[17] M. Burkardt, Phys. Rev. D 69, 091501 (2004).

[18] U. D’Alesio, F. Murgia, and C. Pisano, J. High Energy Phys. 09 (2015) 119.

[19] E. C. Aschenauer, U. D’Alesio, and F. Murgia, Eur. Phys. J. A 52, 156 (2016).

[20] C. Adolph et al. (COMPASS Collaboration), Phys. Lett. B 772, 854 (2017).

[21] Z. Lu and B.-Q. Ma, Phys. Rev. D 94, 094022 (2016).

[22] D. Boer, P. J. Mulders, C. Pisano, and J. Zhou, J. High Energy Phys. 08 (2016) 001.

[23] S. J. Brodsky, F. Fleuret, C. Hadjidakis, and J. P. Lansberg, Phys. Rep. 522, 239 (2013).

[24] D. Kikoa, M. G. Echevarria, C. Hadjidakis, J.-P. Lansberg, C. Lorc, L. Massacrier, C. M. Quintans, A. Signori, and B. Trzeciak, Few-Body Syst. 58, 139 (2017).
[25] F. Dominguez, B.-W. Xiao, and F. Yuan, Phys. Rev. Lett. 106, 022301 (2011).

[26] D. Boer, S. J. Brodsky, P. J. Mulders, and C. Pisano, Phys. Rev. Lett. 106, 132001 (2011).

[27] T. Burton, in Proceedings of the 20th International Workshop on Deep-Inelastic Scattering and Related Subjects (DIS 2012), University of Bonn, 2012 (DESY-PROC-201202), p. 377.

[28] A. Bacchetta, U. D'Alesio, M. Diehl, and C. A. Miller, Phys. Rev. D 70, 117504 (2004).

[29] P. J. Mulders and R. D. Tangerman, Nucl. Phys. B461, 197 (1996); B484, 538(E) (1997).

[30] F. Dominguez, C. Marquet, B.-W. Xiao, and F. Yuan, Phys. Rev. D 83, 105005 (2011).

[31] L. Zheng, E. C. Aschenauer, J. H. Lee, and B.-W. Xiao, Phys. Rev. D 89, 074037 (2014).

[32] T. Sjostrand, S. Mrenna, and P. Z. Skands, J. High Energy Phys. 05 (2006) 026.

[33] C. Alexa et al. (H1 Collaboration), Eur. Phys. J. C 73, 2406 (2013).

[34] F. D. Aaron et al. (H1 Collaboration), Eur. Phys. J. C 71, 1769 (2011); 72, 2252(E) (2012).

[35] A. Airapetian et al. (HERMES Collaboration), J. High Energy Phys. 08 (2010) 130.

[36] C. Adolph et al. (COMPASS Collaboration), Phys. Lett. B 717, 383 (2012).

[37] M. Anselmino, M. Boglione, U. D’Alesio, F. Murgia, and A. Prokudin, J. High Energy Phys. 04 (2017) 046.

[38] M. Anselmino, M. Boglione, U. D'Alesio, E. Leader, and F. Murgia, Phys. Rev. D 70, 074025 (2004).

[39] J. Collins and T. Rogers, Phys. Rev. D 91, 074020 (2015).

[40] J. C. Collins, D. E. Soper, and G. F. Sterman, Nucl. Phys. B250, 199 (1985).

[41] Z.-B. Kang, B.-W. Xiao, and F. Yuan, Phys. Rev. Lett. 107, 152002 (2011).

[42] M. G. Echevarria, A. Idilbi, Z.-B. Kang, and I. Vitev, Phys. Rev. D 89, 074013 (2014).

[43] M. Anselmino, M. Boglione, and S. Melis, Phys. Rev. D 86, 014028 (2012).

[44] G. Bunce, N. Saito, J. Soffer, and W. Vogelsang, Annu. Rev. Nucl. Part. Sci. 50, 525 (2000).

[45] E. C. Aschenauer et al., arXiv:1409.1633.

[46] U. D'Alesio, F. Murgia, C. Pisano, and P. Taels, Phys. Rev. D 96, 036011 (2017). 